\title{
EFFECT OF THE PROPOSED TRAIIING PROGRAM ON SOME PHYSIOLOGICAL AND BODY COMPOSTTION VARIABLES OF UNDER 20 YEARS OLD MALE FOOTBALL PLAYERS
}

\author{
Talha Khanafdl Omar \\ Address for corpespondence: \\ Talha Khanafdl Omar \\ School of Physical Education and Sports, Faculty of Education, Soran University \\ Delzyan Campus, 44008, Soran, Erbil, Kurdistan, Iraq \\ E-mail: talha.omar@pe.soran.edu.iq
}

ORCID: 0000-0003-3279-5736. School of Physical Education and Sports, Faculty of Education, Soran University, Soran, Kurdistan

\begin{abstract}
Ahstract The purpose of the study was to find out the effect of the proposed training program on some physiological and body composition variables of 24 volunteered youth male football players who under 20 years old (U20, age: 16-19 years, mean age: $17.3 \pm 1.0$ years, playing for last 4-6 years). Those 24 players were divided into 2 groups, 12 players (experimental group), 12 players (control group). The training program consisted of aerobic training, anaerobic training, recreational game and practice football game. Data was collected at zero level (pre-test), in the mid of the program (mid-test) and at the end of program (posttest). For analysing data, repeated measures were used. In the experimental group a significant increase $(P<0.05)$ in number of shuttles, skeletal muscle, and resting metabolism and a significant decrease $(P<0.05)$ in body fat have been noted in (midtest) and (post-test) when compared to (pre-test). However, a decrease was noted in body mass index, blood lactate, maximal heart rate, and visceral fat, but not to a significantly different level. In the control group negative significant increase $(P<0.05)$ in body fat and negative significant decrease $(P<0.05)$ in skeletal muscle and resting metabolism have been noted, no significant difference was observed in number of shuttle, body mass index, blood lactate, maximum heart rate and visceral fat. This study would provide useful information for training and exercise physiology and it may have a beneficial impact on health.
\end{abstract}

Key Worlds aerobic training, anaerobic training, blood lactate, body composition, football player

\section{Introduction}

Football is unarguably the world's most popular sport game. A common aspect of this sport is the necessity of teamwork to complement individual skills. In order to adapt to technical evolution within the game, players have to meet the physical demands required. To achieve the best possible performance, training has to be formulated according to the principles of periodization (Bompa, Carrera, 2005). The training induced changes have been observed in various physiological, biochemical and body composition parameters and can be attributed to appropriate load dynamics (Hoff, 2005; Reilly, 2005). As the players have to cover a big area in the ground during 
attack and defense, the game demands high aerobic fitness (Reilly, 2005; Gacesa, Barak, Grujic, 2009; Miller et al., 2007).

A high number of accelerations and decelerations, associated with a large number of changes in the direction of play create an additional load to muscles of football players, which indicates a high need for both the aerobic and anaerobic energy delivery pathways (Miller et al., 2007).

Practicing sports, especially aerobic training, has been proposed to be an effective mechanism for cardiovascular protection, however its effectiveness depends on many factors, including age, sex, body composition and nutrition, as well as training duration and intensity (Ounis et al., 2008; Kim, Kim, 2012; Du Preez, 2013).

During aerobic exercise, the demand for oxygen increases at the working muscle, so an optimum level of hemoglobin is required to perform at the highest level with high intensity. As football performance depends very much on the aerobic component of an athlete, the players need to maintain a normal hemoglobin level to optimize performance (Urhausen, Kindermann, 2002).

Recent studies have identified the anaerobic threshold during resistance exercise, allowing determination of the critical metabolic and cardiovascular changes during this type of exercise generally, the anaerobic threshold in resistance exercise occurs at the low-moderate intensity with high number of repetitions (Simoes et al., 2010).

This study focused on the football players as the game is the most popular and widely played all over the world. The physiological and body composition variables have important roles in the evaluation of training and for assessment of the players' health as well as their metabolic and cardiovascular status. There are insufficient studies in Kurdistan region-Iraq, in this aspect particularly among football players under 20 years of age. In view of the above, the present study has been done.

\section{Material and methods}

\section{Method of the Study}

Experimental method was used for this study. Both groups performed the tests, that is, pre-test, mid-test and post-test, however only the experimental group participated in the training. This is presented below

$\begin{array}{lccccc}\text { Group } & \text { pre-test } & \text { training } & \text { mid-test } & \text { training } & \text { post-test } \\ \text { Experimental } & 0 & \mathrm{X} & 0 & \mathrm{X} & 0 \\ \text { Control } & 0 & & 0 & & 0\end{array}$

\section{Subjects}

All the male football players in Harir Youth and Sports Centre of Erbil city between the age 16 to 19 years were the population for the study. The total population is 69 players of Harir Youth and sports Centre. 24 male football players in the age group of under 20 years of age were intentionally chosen to participate in this study. The participants were divided into two groups, 12 players (experimental group) (XG), and 12 players (control group) (CG). 


\section{Training}

\section{Administration of training program}

To begin the program, the researcher calculated maximum heart rate of each player using the formula: (220 - age $=\mathbf{H R m a x}$ ), then adjusted for each player their heart rates to 60 and $80 \%$ intensity for running pace for aerobic training and anaerobic training respectively. Aerobic training consisted of 3 minutes of running with $60 \%$ intensity heart rate beat and one minute rest repeated 5 times. Anaerobic training consisted of sprint 30 meter dash and sprint 50 meter dash. Each player sprint with intensity $80 \%$ heart rate beat, sprint 30 meter dash and coming back to the start point and repeated this training for 5 times. Another anaerobic training sprint 50 meter dash and coming back to the start point and repeated this training for 5 times. The training was given in the evening time for one hour for the first two weeks and then 10 minutes, which were increased after every two weeks and in the last two weeks the training duration was 110 minutes in a day. The training program was conducted to all the players of the experimental group on every Monday, Wednesday and Friday alternately for 3 months.

Table 1. General schedule of 12 weeks of training program

\begin{tabular}{|c|c|c|c|c|c|c|c|c|c|c|c|c|}
\hline \multicolumn{13}{|c|}{ Macro plan } \\
\hline Month & \multicolumn{4}{|c|}{ October } & \multicolumn{4}{|c|}{ November } & \multicolumn{4}{|c|}{ December } \\
\hline & \multicolumn{4}{|c|}{ Preparatory phase } & \multicolumn{4}{|c|}{ Preparatory phase } & \multirow{2}{*}{\multicolumn{4}{|c|}{$\begin{array}{l}\text { Pre-competition phase } \\
\text { III }\end{array}$}} \\
\hline Period \& meso & \multicolumn{4}{|c|}{1} & \multicolumn{4}{|c|}{$\|$} & & & & \\
\hline Micro/weeks & 1 & 2 & 3 & 4 & 5 & 6 & 7 & 8 & 9 & 10 & 11 & 12 \\
\hline Days & 3 & 3 & 3 & 3 & 3 & 3 & 3 & 3 & 3 & 3 & 3 & 3 \\
\hline Hours & 3 & 3 & 3.5 & 3.5 & 4 & 4 & 4.5 & 4.5 & 5 & 5 & 5.5 & 5.5 \\
\hline \multirow{2}{*}{ Physical fitness } & \multicolumn{4}{|c|}{10 hours 12 minutes } & \multicolumn{4}{|c|}{10 hours 12 minutes } & \multicolumn{4}{|c|}{10 hours 12 minutes } \\
\hline & \multicolumn{4}{|c|}{ Aerobic and anaerobic training } & \multicolumn{4}{|c|}{ Aerobic and anaerobic training } & \multicolumn{4}{|c|}{ Aerobic and anaerobic training } \\
\hline Recreational game \& Practice game & \multicolumn{4}{|c|}{2 hours 48 minutes } & \multicolumn{4}{|c|}{6 hours 48 minutes } & \multicolumn{4}{|c|}{10 hours 48 minutes } \\
\hline
\end{tabular}

\section{Measurement of physiological variables}

The maximum heart rate was measured after 3 minutes long 20 meter shuttle run by using (polar RS 400 heart rate monitor USA model), and lactate blood level was measured after 3 minutes long 20 meter shuttle run by using lactate pro 2 test meter and lactate pro 2 strips, (Arkary model, Japan). Cardio-respiratory endurance was indirectly measured during 3 minutes long 20 meter shuttle run by counting the number of shuttles, cone, stop watch and clapper were used for this test. The shuttle run test was administered outdoor on the artificial football surface.

\section{Measuruement of hody composition variables}

In the morning as soon as the selected subjects awoke, body mass index, body fat percentage, skeletal muscle percentage, resting metabolism and visceral fat level, were measured using the bioelectrical impedance method by using a full body sensor and a body composition monitor and scale (Omron machine model HBF-514, USA). 


\section{Statistical procedure employed}

For testing the statistical significance difference between the pre-test, mid-test, and post-test repeated measures were performed and independent sample t-test was used to find out the significant difference between pre-test, mid-test and post-test of both groups at each level of the tests. The level of significance was kept $P=0.05$ in order to test the null hypothesis and alternative hypothesis. SPSS (Version 23.) was used for analyzing data.

\section{Results}

Tahle 2. Effect of Training on Physiological variables of U-20 years old male football Players

\begin{tabular}{lccccccc}
\hline \multicolumn{1}{c}{ Variables } & Unit & Groups & Pre-test & Mid-test & Post-test & P & $F$ \\
\hline \multirow{2}{*}{ Number of shuttles } & \multirow{2}{*}{ shuttles } & XG & $27.8 \pm 1.4$ & $29.5 \pm 1.6$ & $31.1 \pm 1.5$ & $0.01^{*}$ & 15.94 \\
& & CG & $27.5 \pm 1.3$ & $28.3 \pm 1.5$ & $28.7 \pm 1.0$ & 0.07 & 11.46 \\
\hline \multirow{2}{*}{ Blood lactate } & \multirow{2}{*}{ Ml } & XG & $12.2 \pm 5.9$ & $9.8 \pm 3.7$ & $9.1 \pm 4.5$ & 0.44 & 4.07 \\
& & CG & $11.8 \pm 5.6$ & $11.6 \pm 2.3$ & $10.6 \pm 3.9$ & 0.32 & 0.27 \\
\hline \multirow{2}{*}{ HRmax } & \multirow{2}{*}{ BPM } & XG & $182.0 \pm 5.5$ & $176.8 \pm 7.8$ & $176.6 \pm 6.6$ & 0.74 & 14.37 \\
& & CG & $183.4 \pm 6.7$ & $183.7 \pm 9.9$ & $182.0 \pm 7.4$ & 0.37 & 0.32 \\
\hline
\end{tabular}

Repeated measures (Post Hoc) test was performed. Each value represents mean $\pm S D . N=24$, each group $=12$. Computed using alpha $=0.05 .{ }^{*}$ Significance difference when compared to post-test. XG - Experimental group. CG - Control group.

As stated in 1 there is a significant difference between pre-test and post-test on the number of shuttles of experimental $(X G)$ group because $P$ value is $(0.01)$, and there is no significant difference between pre-test and post-test on the number of shuttles of control (CG) group because $P$ value is $(0.07)$. Also there is no significant difference between pre-test and post-test on the blood lactate level of experimental $(X G)$ group because $P$ value is $(0.44)$, also there is no significant difference between pre-test and post-test on the blood lactate level of control (CG) group because $P$ value is (0.32). Also 3 there is no significant difference between pre-test and post-test on maximum heart rate of experimental $(X G)$ group because $P$ value is $(0.74)$, also there is no significant difference between pre-test and post-test on maximum heart rate of control (CG) group because $P$ value is $(0.37)$.

Table 3. Effect of Training on body composition variables of U-20 years old male football Players

\begin{tabular}{|c|c|c|c|c|c|c|c|}
\hline Variables & Units & Groups & Pre-test & Mid-test & Post-test & $P$ & $\mathrm{~F}$ \\
\hline \multirow{2}{*}{ BMl } & \multirow{2}{*}{$\mathrm{kg} / \mathrm{m}^{2}$} & $X G$ & $19.9 \pm 1.9$ & $19.5 \pm 1.8$ & $19.2 \pm 1.6$ & 0.20 & 16.40 \\
\hline & & CG & $19.8 \pm 2.3$ & $20.2 \pm 2.8$ & $20.6 \pm 2.9$ & 0.16 & 13.00 \\
\hline \multirow{2}{*}{ Body fat } & \multirow{2}{*}{$\mathrm{mm}$} & $X G$ & $11.9 \pm 4.6$ & $10.9 \pm 4.4$ & $10.6 \pm 4.5$ & $0.01^{*}$ & 200.12 \\
\hline & & CG & $11.8 \pm 5.7$ & $12.7 \pm 5.6$ & $13.4 \pm 5.9$ & $0.03^{*}$ & 35.94 \\
\hline \multirow{2}{*}{ Skeletal muscle } & \multirow{2}{*}{$\mathrm{kg}$} & $X G$ & $45.1 \pm 2.4$ & $45.8 \pm 2.2$ & $46.2 \pm 2.2$ & $0.05^{*}$ & 91.33 \\
\hline & & CG & $45.7 \pm 3.6$ & $44.3 \pm 3.4$ & $43.6 \pm 3.4$ & $0.06^{*}$ & 28.94 \\
\hline \multirow{2}{*}{ Resting metabolism } & \multirow{2}{*}{ k.calori } & $X G$ & $1475 \pm 87.7$ & $1483 \pm 87.6$ & $1505 \pm 91.1$ & $0.01^{*}$ & 26.78 \\
\hline & & CG & $1481 \pm 105.4$ & $1469 \pm 84.3$ & $1466 \pm 84.3$ & $0.01^{*}$ & 0.54 \\
\hline \multirow{2}{*}{ Visceral fat } & \multirow{2}{*}{$\mathrm{cm}^{3}$} & $X G$ & $2.5 \pm 1.6$ & $2.0 \pm 1.3$ & $1.6 \pm 1.3$ & 0.18 & 8.87 \\
\hline & & CG & $2.5 \pm 2.1$ & $3.0 \pm 2.2$ & $3.3 \pm 2.4$ & 0.19 & 8.55 \\
\hline
\end{tabular}

Repeted measures (Post Hoc) test was performed. Each value represents mean \pm SD. $N=24$, each group $=12$. Computed using alpha $=0.05$.

* significance differenace when compared to post-test. XG -Experimental group. CG - Control group. 
As revealed in Table 3, there was not significant difference between pre-test and post-test on body mass index of experimental $(X G)$ group because $P$ value is $(0.20)$, and there is no significant difference between pre-test and post-test on body mass index of control (CG) group because $P$ value is $(0.16)$. Also there was a significant difference between pre-test and post-test on Body fat of experimental $(X G)$ group because $P$ value is $(0.01)$, and there is negative significant difference between pre-test and post-test on the Body fat of control (CG) group because $P$ value is (0.03). Also there is a significant difference between pre-test and post-test on skeletal muscle of experimental (XG) group because $P$ value is $(0.05)$, also there is negative significant difference between pre-test and post-test on skeletal muscle of control (CG) group because $P$ value is (0.006). Also there is a significant difference between pre-test and post-test on resting metabolism of experimental $(X G)$ group because $P$ value is $(0.01)$, also there is a significant difference between pre-test and post-test on Resting metabolism of control (CG) group because $P$ value is (0.01). Also there is no significant difference between pre-test and post-test on visceral fat of experimental $(X G)$ group because $P$ value is (0.18), there is no significant difference between pre-test and post-test on visceral fat of control (CG) group because $P$ value is (0.19).

\section{Discussion}

In the present study, it was observed that subjects under 20 years of age were more interested in the recreational games and practice football matches more than the aerobic and anaerobic training. After analyzing and interpretation of the data, the study revealed a significant increase $(P=0.01)$ in number of shuttles of the experimental group (XG). The increase in the number of shuttles during 3 minutes after training reflects improved cardio-respiratory endurance capacity and aerobic capacity. Cardio-respiratory endurance and aerobic capacity certainly play an important role in football game and has a major influence on technical performance and tactical choices (Reilly, 2005; Mc Ardle, Katch, Katch, 2006; Wilmore, Costill, 2005). The aerobic endurance training enhances the activity of the cardiovascular system as well as the developed oxidative capacity of the skeletal muscles, and thus oxygen delivery to the working muscle is increased. This was accepted as the main reason for the elevation of aerobic capacity after a training program (McArdle et al., 2006; Wilmore, Costill, 2005). A similar observation was noted by other researchers (Hoff, 2005; Reilly, 2005; Miller et al., 2007). On the other hand, no significant difference $(P=0.07)$ in number of shuttles of control group $(C G)$,

During the exercise of increasing intensity there is a rise in blood lactate concentration resulting from increased glycogenolysis. This increase in blood lactate concentration has been interpreted as a reflection of the onset of hypoxia in skeletal muscles and the exercise intensity at which anaerobic metabolism generates ATP is known as the anaerobic threshold (McArdle et al., 2006; Wilmore, Costill, 2005). Increased activity of the muscle form of lactate dehydrogenase and sensitivity to increasing metabolic acidosis. A major effect of endurance training is thought to enhance lactate clearance (Ali et al., 2012). The experimental group (XG) of this study showed a decrease of blood lactate level, but no statistical significant difference $(P=0.44)$ observed Another research which was done by (Manna, Khanna, 2013) in the study "effect of training on selected biochemical variables of elite male swimmers", they are reported significant decreased $(P<0.05)$ in peak blood lactate levels of the swimmers. It may be due to the difference in the type of sports and games has on the effect of blood lactate level, and controlling blood lactate level not easy for researchers in the day of testing. It should also be noted that activity of daily living has an effect on the blood lactate level. Also the control group (CG) showed that no significant difference was observed because $P$ value is $(0.44)$. 
Heart rate increases with an increase in work intensity and shows a linear relationship with work rate (Astrand, Rodhal, 1986). The highest rate at which the heart can beat is the maximal heart rate (McArdle et al., 2006; Wilmore, Costill, 2005). The experimental group of this study showed a decrease of blood lactate level, but no statistical significant difference $(P=0.74)$ observed, Another research finding showed similar results which was done by (Manna et al., 2012) in the study "Effect of training on anthropometric, physiological and biochemical variables of U-19 volleyball players". It has been seen that short term exercise has no significant effect on maximum heart rate (Mc Ardle et al., 2010; Wilmore, Costill, 2005). Also in control group (CG) no significant difference between pre-test and post-test in maximum heart rate was observed because value is $(P=0.37)$.

Body mass index has significant impact on football teams (Hoff, 2005; Johnson, Doherty, Freemont, 2009) Body mass is a considerable factor in football since body contact is essential in this game (Hoff, 2005; Johnson et al., 2009). The experimental group of this study showed that there was no significant difference $(P=0.20)$ observed in body mass index of the football players after the post-test. It may be due to the shorter duration of the training. It has been reported that short term exercise training has no significant effect on body mass of the sports persons (McArdle et al., 2006; Wilmore, Costill, 2005). Another research finding showed similar results which was done by (Manna, Khanna, Dhara, 2009) in the study "Training induced changes on physiological and biochemical variables of young Indian field hockey players". Also In other side with a control group of the present study observed no significant difference $(P=0.16)$ has been observed in body mass index of the football players after the post-test.

The percentage of body fat plays an important role in the assessment of physical fitness of the soccer players (Hoff, 2005; Ostojic, 2003). A low-body fat may improve athletic performance by improving the strength to weight ratio (Reilly, 2005; McArdle et al., 2006; Wilmore, Costill, 2005). Excess body fat adds to the load without contributing to the body's force-producing capacity (Reilly, 2005; McArdle et al., 2006; Wilmore, Costill, 2005). The experimental group of this study showed that significant reduction $(P=0.01)$ in percent body fat has been noted among the players after the training program. The possible reason of reduction of body fat is exercise training which increases greater utilization of fat for energetic (McArdle et al., 2006; Wilmore, Costill, 2005). Similar findings were also noted by other research groups who studied on soccer players and reported that percent body fat decreased significantly during preparatory and competitive phase of training when compared to baseline data (Reilly, 2005). On the other hand, in a control group of the present study observed negative significant increase difference $(P=0.03)$ observed.

A recent research finding which was done by (Kirkendall, Garret, 1998) in the study "The Effects of Aging and Training on Skeletal Muscle" reported that Endurance training can improve the aerobic capacity of muscle, and resistance training can improve central nervous system recruitment of muscle and increase muscle mass. Therefore, physical activity throughout life is encouraged to prevent much of the age-related impact on skeletal muscle. The experimental group of this study showed a positive significant increase $(P=0.05)$ in skeletal muscle. Whilst the control group of the respective study observed a negative significant decrease $(P=0.006)$ in skeletal muscle. Also (Kirkendall, Garret, 1998) in the studied "The Effects of Aging and Training on Skeletal Muscle" reported Aging results in a gradual loss of muscle function, the typical adult will lose muscle mass with age; the loss varies according to gender.

Another recent research which was done by (Lemmer et al., 2001) in the study "Effect of strength training on resting metabolic rate and physical activity: age and gender comparisons" reported absolute significant increase $(P<0.01)$ in resting metabolic rate after training in young age. In the experimental group of this study showed that 
a significant increase $(P=0.01)$ in resting metabolism. Where as the control group of the present study observed a significant decrease $(P=0.01)$ in resting metabolism.

The experimental group of this study showed a decrease in visceral fat, but statistically no significant difference $(P=0.18)$ was observed. Another research which was done by (Giannaki, Aphamis, Sakkis, Hadjicharalambous, 2015) in the study "Eight weeks of a combination of high intensity interval training and conventional training reduce visceral adiposity and improve physical fitness: a group-based intervention" reported both exercise programs were effective in reducing total body fat and visceral adiposity $(p<0.05)$, It may be due to the different type intensity in training has an effect on visceral fat. Also in a control group of the present study showed that increase visceral fat, but statistically no significant difference $(P=0.19)$ observed.

\section{Conclusion}

Cardio-respiratory endurance is an important part of overall physical fitness. An increase in the number of shuttles during 3 minutes after training reflects improved cardio-respiratory endurance capacity and aerobic capacity, it will help the physical educator and coaches to apply technical and tactical plan faster during the match and competition, especially this finding is important for midfield football players to do more about running in tactical plans in the middle of the ground. Regular measurement of blood lactate level and maximum heart rate are important to determine optimum intensity in the training and in the different phases of training. Because of training program Reduce of blood lactate in this research. As we can see the results of current research reduction occurs of body fat, visceral fat. It is important for player's performance as well as it helps in minimizing the risk factor for cardiovascular disease among the players, especially for older adults. The results of skeletal muscle of this research increase, building and increasing skeletal muscle are much important to prevent injuries. Measurement of resting metabolic rate is important for determining the optimum number of calories for players to burn it at rest, during training, and vital function of the human body. As the studies on football players are limited in this aspect in Kurdistan region-Iraq, the data of the present study can be a handy tool and can act as a frame of reference for monitoring of training of football players particularly of under 20 years old group. This would enable the coaches to assess the current status of an athlete and the degree of training, adaptability and provide an opportunity to modify the training schedule accordingly to achieve the desired performance.

\section{References}

Ali, R.S., Koushkie, J.M., Asadmanesh, A., Salesi, M. (2012). Influence of massage, active and passive recovery on swimming performance and blood lactate. The Journal of sports medicine and physical fitness, 52 (2), 122-127.

Åstrand, P.O., Rodahl, K., Dahl, H.A., Strømme, S.B. (2003). Textbook of work physiology: physiological bases of exercise. Human Kinetics.

Bompa, T.O., Carrera, M. (2005). Periodization training for sports (Vol. 3). Champaign (IL): Human Kinetics.

du Preez, C. (2013). A 10-week aerobic exercise program reduces cardiometabolic disease risk in overweight/obese female African university students. Ethnicity \& disease, 23, 143.

Gacesa, J.Z.P., Barak, O.F., Grujic, N.G. (2009). Maximal anaerobic power test in athletes of different sport disciplines. The Journal of Strength \& Conditioning Research, 23 (3), 751-755.

Giannaki, C.D., Aphamis, G., Sakkis, P., Hadjicharalambous, M. (2015). Eight weeks of a combination of high intensity interval training and conventional training reduce visceral adiposity and improve physical fitness: a group-based intervention. The Journal of Sports Medicine and Physical Fitness.

Hoff, J. (2005). Training and testing physical capacities of elite soccer players. Journal of sports sciences, 23 (6), 573-582. 
Johnson, A., Doherty, P.J., Freemont, A. (2009). Investigation of growth, development, and factors associated with injury in elite schoolboy footballers: prospective study. Bmj, 338, b490.

Kenney, W.L., Wilmore, J., Costill, D. (1999). Physiology of sport and exercise with web study guide. Human Kinetics.

Kim, J.W., Kim, D.Y. (2012). Effects of aerobic exercise training on serum sex hormone binding globulin, body fat index, and metabolic syndrome factors in obese postmenopausal women. Metabolic Syndrome and Related Disorders, 10 (6), 452-457.

Kirkendall, D.T., Garrett, W.E. (1998). The effects of aging and training on skeletal muscle. The American Journal of Sports Medicine, 26 (4), 598-602.

Lemmer, J.T., Ivey, F.M., Ryan, A.S., Martel, G.F., Hurlbut, D.E., Metter, J.E., Fozard, J.L., Fleg, J.L., Hurley, B.F. (2001). Effect of strength training on resting metabolic rate and physical activity: age and gender comparisons. Medicine and Science in Sports and Exercise, 33 (4), 532-541.

Manna, I., Khanna, G.L. (2013). Effect of Training on Selected Biochemical Variables of Elite Male Swimmers. American Journal of Sports Science and Medicine, 1 (2), 13-16.

Manna, I., Khanna, G.L., Dhara, P.C. (2009). Training induced changes on physiological and biochemical variables of young Indian field hockey players. Biology of Sport, 26 (1), 33.

McArdle, W.D., Katch, F.I., Katch, V.L. (2006). Essentials of exercise physiology. Lippincott Williams \& Wilkins.

McArdle, W.D., Katch, F.I., Katch, V.L. (2010). Exercise physiology: nutrition, energy, and human performance. Lippincott Williams \& Wilkins.

Miller, T.A., Thierry-Aguilera, R., Congleton, J.J., Amendola, A.A., Clark, M.J., Crouse, S.F., Marin, S.V., Jenkins, O.C. (2007). SEASONAL CHANGES IN [Latin capital $V$ with dot above] O2max AMONG DIVISION 1A COLLEGIATE WOMEN SOCCER PLAYERS. The Journal of Strength \& Conditioning Research, 21 (1), 48-51.

Ostojic, S.M. (2003). Seasonal alterations in body composition and sprint performance of elite soccer players. Journal of Exercise Physiology, 6 (3), 11-14.

Ounis, O.B., Elloumi, M., Chiekh, I.B., Zbidi, A., Amri, M., Lac, G., Tabka, Z. (2008). Effects of two-month physical-endurance and dietrestriction programs on lipid profiles and insulin resistance in obese adolescent boys. Diabetes \& Metabolism, 34 (6), 595-600.

Reilly, T. (2005). An ergonomics model of the soccer training process. Journal of Sports Sciences, 23 (6), 561-572.

Simões, R.P., Mendes, R.G., Castello, V., Machado, H.G., Almeida, L.B., Baldissera, V., Catai, A.M., Arena, R., Borghi-Silva, A. (2010). Heart-rate variability and blood-lactate threshold interaction during progressive resistance exercise in healthy older men. The Journal of Strength \& Conditioning Research, 24 (5), 1313-1320.

Urhausen, A., Kindermann, W. (2002). Diagnosis of overtraining. Sports Medicine, 32 (2), 95-102.

Cite this anticle aS: Omar, T.K. (2021). Effect of the Proposed Training Program on some Physiological and Body Composition Variables of under 20 Years Old Male Football Players. Central European Journal of Sport Sciences and Medicine, 2 (34), 97-104. DOI: 10.18276/cej.2021.2-09. 